\title{
Tumor fibroso solitario de glándula suprarrenal izquierda asociado con síndrome de Doege-Potter: reporte de caso
}

\author{
Solitary fibrous tumor of the left adrenal gland associated with Doege-Potter syndrome. \\ Case report \\ Joaquín D. Campista-Jácquez ${ }^{1,2 *}$ y Héctor R. Romero-Talamás ${ }^{3}$ \\ ${ }^{1}$ Servicio Social; ${ }^{2}$ Departamento de Cirugía General; ${ }^{3}$ Servicio de Enseñanza. Hospital Ángeles Chihuahua, Chihuahua, Chihuahua, México
}

\begin{abstract}
Resumen
Se presenta el caso de un varón de 71 años con antecedente de diabetes mellitus tipo 2 que manifiesta dolor abdominal, pérdida de peso, disnea de pequeños esfuerzos, debilidad muscular y aumento de volumen subcostal izquierdo. Asociado a esto desarrolló episodios frecuentes de síncope, diaforesis y sudoraciones nocturnas, durante los cuales se documentaron cifras de glucemia < $50 \mathrm{mg} / \mathrm{dl}$ y mejoría tras la administración de bebidas hipercalóricas o dextrosa intravenosa. La tomografía computarizada abdominal reveló la presencia de una tumoración de aproximadamente $20 \mathrm{~cm}$ dependiente de la glándula suprarrenal izquierda. Se obtuvo diagnóstico histopatológico de tumor fibroso solitario retroperitoneal confirmado por inmunohistoquímica.
\end{abstract}

Palabras clave: Tumor fibroso solitario. Glándula suprarrenal. Síndrome de Doege-Potter. Hipoglucemia.

\begin{abstract}
We present the case of a 71-year-old man with a history of type 2 diabetes mellitus manifesting abdominal pain, weight loss, dyspnea on small efforts, muscle weakness, and left subcostal swelling. Associated with this, he developed frequent episodes of syncope, diaphoresis, and night sweats, during which blood glucose levels below $50 \mathrm{mg} / \mathrm{dL}$ and improvement were documented after the administration of hypercaloric beverages or intravenous dextrose. Abdominal computed tomography revealed the presence of a tumor of approximately $20 \mathrm{~cm}$ dependent on the left adrenal gland. Histopathological diagnosis of a solitary retroperitoneal fibrous tumor was obtained, confirmed by immunohistochemistry.
\end{abstract}

Key words: Solitary fibrous tumor. Adrenal gland. Doege-Potter syndrome. Hypoglycemia.

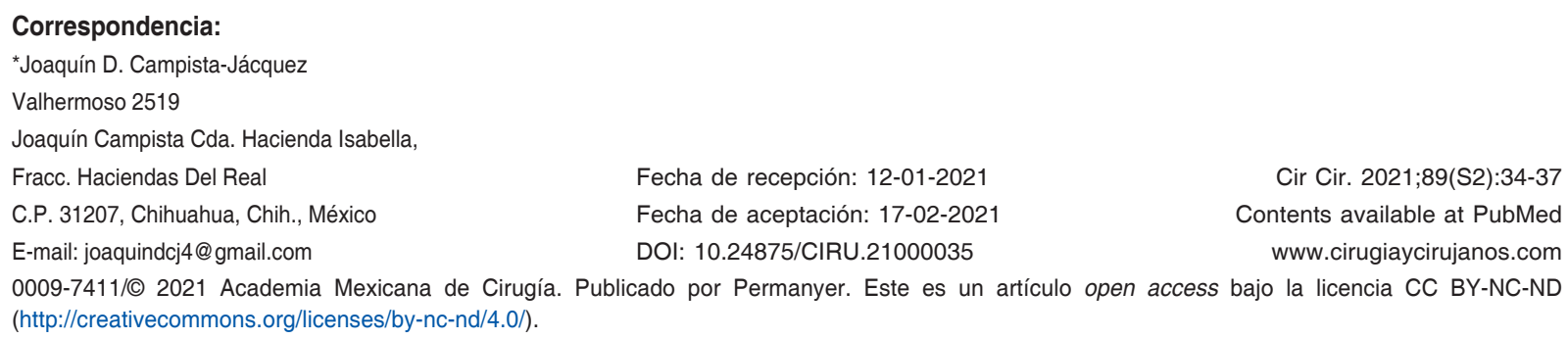

Cir Cir. 2021:89(S2):34-37 Contents available at PubMed www.cirugiaycirujanos.com 0009-7411/@ 2021 Academia Mexicana de Cirugía. Publicado por Permanyer. Este es un artículo open access bajo la licencia CC BY-NC-ND (http://creativecommons.org/licenses/by-nc-nd/4.0/). 


\section{Introducción}

Los tumores fibrosos solitarios son un grupo de neoplasias poco frecuentes de origen mesenquimal que representan el $2 \%$ de todas las tumoraciones de tejidos blandos'. Su localización más frecuente es en la pleura, las meninges o las extremidades; sin embargo, pueden encontrarse en cualquier región anatómica asociados a tejidos blandos y vísceras. Del total de estos tumores, únicamente menos del $5 \%$ se manifiestan con episodios de hipoglucemia ${ }^{2,3}$, síndrome paraneoplásico descrito por primera vez por Doege y Potter en $1930^{4,5}$.

Presentamos un caso de tumor fibroso solitario dependiente de la glándula suprarrenal izquierda asociado a síndrome de Doege-Potter.

\section{Caso clínico}

Varón de 71 años con antecedente de hipertensión arterial sistémica de 15 años de evolución, controlado con valsartán y amlodipino, y diabetes mellitus tipo 2 de 15 años de evolución, manejada con sitagliptina y metformina, que fueron suspendidas debido a los episodios de síncope y diaforesis atribuidos a hipoglucemia. Debido a esto acude con su médico por presentar, desde hace 2 meses, episodios de pérdida del estado de alerta en múltiples ocasiones, acompañados de diaforesis, polidipsia, ansiedad y sudoraciones nocturnas. Durante los episodios se documentaron cifras de glucemia de alrededor de $40 \mathrm{mg} / \mathrm{dl}$ que respondieron al consumo de alimentos hipercalóricos. Aunado a esto, manifestó disnea de pequeños esfuerzos, distensión y dolor abdominal, mareos, debilidad muscular y pérdida involuntaria de peso de aproximadamente $12 \mathrm{~kg}$ en el año previo. Se realizó una tomografía computarizada de abdomen con contraste intravenoso, en la que se observó una tumoración dependiente de la glándula suprarrenal izquierda de $22 \times 17 \times 20 \mathrm{~cm}$, de aspecto sólido, ejerciendo desplazamiento del bazo, el estómago y el páncreas, así como sobre el riñón correspondiente (Fig. 1). No se observaron datos sugestivos de infiltración periférica. Las pruebas de laboratorio realizadas antes de la intervención quirúrgica mostraron glucemia $44 \mathrm{mg} / \mathrm{dl}$ (76-106), insulina sérica $<1.0 \mathrm{UI} / \mathrm{ml}$ (2.3-26), péptido C $0.2 \mathrm{mg} / \mathrm{ml}$ (0.78-5.19), factor de crecimiento similar a la insulina tipo I (IGF-I) $52.80 \mathrm{ng} / \mathrm{ml}$ (35.1-216), factor de crecimiento similar a la insulina tipo II (IGF-II) $220 \mathrm{ng} / \mathrm{ml}$ (267-616) y proteína 3 de unión al factor de crecimiento similar a la

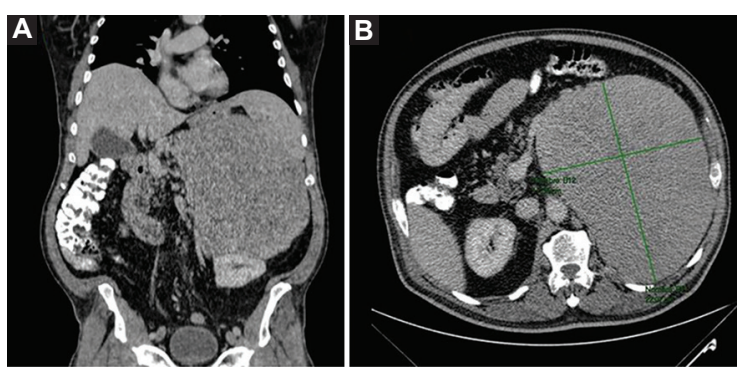

Figura 1. Tomografía computarizada de abdomen en la que se observa una tumoración en el polo superior del riñón izquierdo. A: corte coronal. B: corte axial.

insulina (IGFBP3) $1.1 \mathrm{mg} / \mathrm{l}(2.5-5-7)$. Se realizó la resección del tumor mediante abordaje abierto, con una incisión subcostal izquierda amplia. No se encontró infiltración de las estructuras circundantes, como la cola del páncreas y el colon transverso. Fue necesario realizar una esplenectomía como parte de la resección en bloque. El tumor extraído pesó $3943 \mathrm{~g}$ y midió 23.7 $\times 20.2 \times 13 \mathrm{~cm}$ (Fig. 2). El diagnóstico de tumor solitario fibroso dependiente de la glándula suprarrenal izquierda fue confirmado mediante inmunohistoquími$\mathrm{ca}$, resultando con tinciones intensamente positivas para vimentina, CD99, BCL-2, STAT6 y CD34. El paciente evolucionó de manera favorable en el posoperatorio sin presentar incidentes, con remisión completa de los episodios de hipoglucemia desde el primer día posterior a la intervención. Fue dado de alta al quinto día de posoperatorio, sin complicaciones.

\section{Discusión}

Los tumores fibrosos solitarios son un grupo de tumores poco frecuentes de tipo mesenquimal originados en los fibroblastos y miofibroblastos que comúnmente se localizan en la pleura visceral, y representan menos del $2 \%$ de todas las masas de tejidos blandos ${ }^{1}$. Su descripción inicial fue realizada en un tumor de origen pleural por Klemperer y Rabin en $1931^{6}$. Estos tumores están categorizados con un potencial biológico intermedio, con bajo riesgo de metástasis y un curso clínico relativamente indolente según la Clasificación de la Organización Mundial de la Salud (OMS) de 20027.

Estas lesiones tienen unas características histológicas que se superponen con las de otros tumores de tejidos blandos. Típicamente se componen de células fusiformes bipolares o células de forma ovoide en un estroma de abundante colágeno y alta vascularización, 


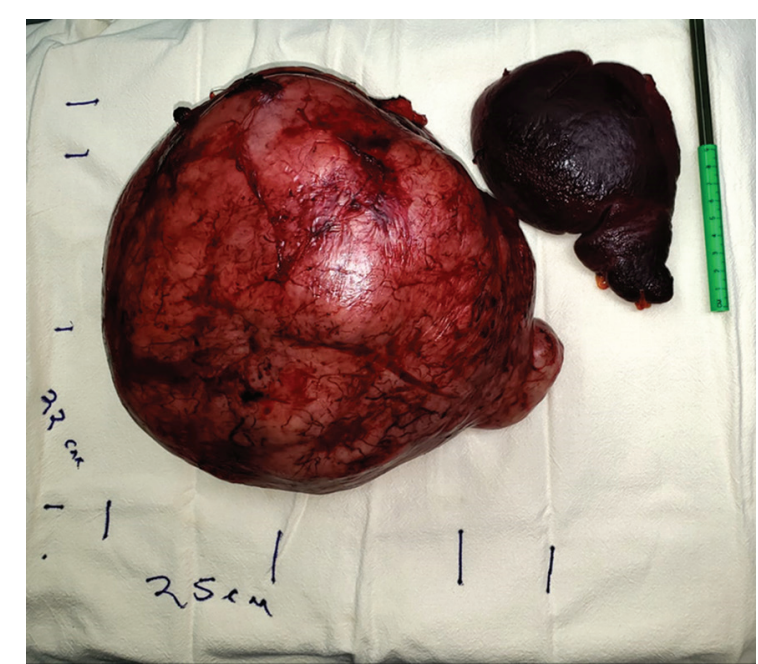

Figura 2. Tumor resecado (a la izquierda) y bazo resecado (a la derecha).

en un patrón similar al de los hemangiopericitomas ${ }^{8}$. En el estudio inmunohistoquímico son positivos para vimentina, CD34, CD99 y BCL-28-10. Adicionalmente se ha demostrado que los tumores fibrosos solitarios se caracterizan por una mutación de reordenamiento de tipo inversión en 12q13-15, generando así la fusión de los genes NAB2-STAT6, y por lo tanto llevando a la sobreexpresión de la proteína STAT6 ${ }^{11,12}$. Actualmente, la OMS los clasifica como tumores de partes blandas ${ }^{7}$.

Los tumores fibrosos solitarios se pueden encontrar en cualquier región anatómica, siendo la localización intratorácica la más común, seguida por la intraabdominal13,14; sin embargo, se ha descrito en otros lugares, como la cabeza, el cuello, el mediastino, la pelvis y la localización retroperitoneal, que es la más infrecuente $\mathrm{t}^{10,15,16}$. Los tumores fibrosos solitarios en el mediastino, el abdomen, el retroperitoneo y la pelvis suelen comportarse de forma más agresiva ${ }^{14}$.

Su distribución es similar entre hombres y mujeres, aparecen en adultos de todas las edades (en general entre la quinta y la sexta décadas de la vida) y son más frecuentes en población caucásica, seguida de la hispánica, la asiática y finalmente la afroamericana ${ }^{17}$.

En ocasiones, los tumores fibrosos solitarios se pueden manifestar en primera instancia con síndromes paraneoplásicos, siendo la hipoglucemia refractaria al tratamiento lo más común. Este fenómeno se describe como hipoglucemia de tumores de células no insulares (NICTH, non-islet cell tumor hypoglyce$\mathrm{mia})^{18}$. Cuando el tumor fibroso solitario se acompaña de hipoglucemias constituye el síndrome de Doege-Potter, descrito por primera vez en $1930^{4,5}$. Ocurre en menos del $5 \%$ de todos los casos y se ve principalmente en tumores pleurales y retroperitoneales de grandes dimensiones ${ }^{2,3}$. El síndrome de Doege-Potter se caracteriza por hipoglucemias refractarias, con valores séricos disminuidos de insulina, péptido $\mathrm{C}$ y hormona del crecimiento, además de unas bajas concentraciones séricas de IGF-I frente a unas cifras de IGF-II que pueden ser normales o elevadas ${ }^{19}$.

La principal causa de este tipo de hipoglucemia es la producción tumoral de IGF-II, un péptido de 7.5 $\mathrm{kDa}^{20-22}$, lo cual resulta en la estimulación de los receptores de insulina (IR-A) y el aumento del uso celular de glucosa. En condiciones normales, el IGF-II circula en un complejo de $150 \mathrm{kDa}$ conformado por IGF-I, IGF-II e IGFBP3, y puede estar asociado a la subunidad ácida lábil$^{23}$. Los IGF son una familia de hormonas peptídicas $y$ factores de crecimiento que incluyen a la insulina, el IGF-I y el IGF-II, y tienen por lo menos un $50 \%$ de los aminoácidos en común con la insulina ${ }^{24}$. La relación entre el IGF-II y la NICTH fue identificada por Daughaday en $1988^{25}$ en una paciente con hipoglucemia y con un fibrosarcoma, cuyos valores expresados de ARNm de IGF-II y de péptido IGF-II eran muy elevados.

En la NICTH, los valores elevados de IGF-II son consecuencia de la sobreexpresión tumoral de ARNm de IGF-II, que además da a lugar a precursores inmaduros de IGF-II, big IGF-II y pro-IGF-II, los cuales también pueden interactuar con los receptores de insulina tisulares $^{26}$. Se ha descrito que la fisiopatología de la NICTH sigue un mecanismo dual: incremento del uso de glucosa celular mediado por IGF-II, big IGF-II o pro-IGF-II, e inhibición de la hormona de crecimiento, disminuyendo la contrarregulación de la hipoglucemia por esta ${ }^{19,26,27}$. No obstante, se ha descrito que el IGF-II se expresa en el $80 \%$ de los tumores fibrosos solitarios aun con valores elevados de ARNm de IGF-II' ${ }^{19}$. Aunque es posible que en estos pacientes las concentraciones séricas de IGF-II estén elevadas, a menudo son normales en cantidad, tal como sucedía en nuestro paciente; sin embargo, los valores de big IGF-II y pro-IGF-II medidos por ensayo de inmunotransferencia son elevados en casos de NICTH, lo que explicaría el origen de la hipoglucemia en los pacientes con valores bajos o normales de IGF-II, o simplemente podría atribuirse al consumo de glucosa por parte del tumor ${ }^{26,28}$.

El tratamiento inicial debe consistir en aumentar el aporte de glucosa oral e intravenosa para evitar la hipoglucemia. Se ha reportado el uso de glucagón, hormona de crecimiento y glucocorticoides ${ }^{19,29}$. El tratamiento quirúrgico continúa siendo el manejo definitivo para esta enfermedad, pero se ha reportado la 
recurrencia hasta 20 años después de la cirugía ${ }^{30}$; en general, la progresión sin metástasis a 5 y 10 años es del $74 \%$ y el $55 \%$, respectivamente, mientras que las tasas de supervivencia específicas de la enfermedad a 5 y 10 años son del $89 \%$ y el $73 \%$. La edad del paciente, el tamaño del tumor y el índice mitótico son predictores tanto del tiempo para la metástasis como para la mortalidad específica por enfermedad, mientras que la necrosis pronosticó solo la metástasis ${ }^{17}$. A pesar de que la mayoría de los tumores fibrosos solitarios suelen ser benignos, entre el $15 \%$ y el $20 \%$ son agresivos y eventualmente recurrentes ${ }^{31}$.

\section{Conclusiones}

El síndrome de Doege-Potter es un síndrome paraneoplásico poco frecuente que se caracteriza por hipoglucemias graves refractarias al tratamiento, acompañado de un tumor fibroso solitario. Se debe tener la sospecha clínica ante un paciente con antecedente de hipoglucemias de difícil control, ya que dicha sospecha nos puede llevar a encontrar la causa subyacente. El tratamiento curativo de los tumores fibrosos solitarios y del síndrome de Doege-Potter continúa siendo la resección quirúrgica, aunque se puede apoyar de manera inicial aumentando la ingesta oral de glucosa o por vía intravenosa.

\section{Responsabilidades éticas}

Protección de personas y animales. Los autores declaran que los procedimientos seguidos se conformaron a las normas éticas del comité de experimentación humana responsable y de acuerdo con la Asociación Médica Mundial y la Declaración de Helsinki.

Confidencialidad de los datos. Los autores declaran que han seguido los protocolos de su centro de trabajo sobre la publicación de datos de pacientes.

Derecho a la privacidad y consentimiento informado. Los autores han obtenido el consentimiento informado de los pacientes y/o sujetos referidos en el artículo. Este documento obra en poder del autor de correspondencia.

\section{Conflicto de intereses}

Los autores declaran que no existe ningún conflicto de intereses relacionado con el artículo.

\section{Bibliografía}

1. Davanzo B, Emerson RE, Lisy M, Koniaris LG, Kays JK. Solitary fibrous tumor. Transl Gastroenterol Hepatol. 2018;3:94.
2. Jang JG, Chung JH, Hong KS, Ahn JH, Lee JY, Jo JH, et al. A case of solitary fibrous pleura tumor associated with severe hypoglycemia: doege-potter syndrome. Tuberc Respir Dis (Seoul). 2015;78:120-4.

3. Meng W, Zhu HH, Li H, Wang G, Wei D, Feng X. Solitary fibrous tumors of the pleura with Doege-Potter syndrome: a case report and three-decade review of the literature. BMC Res Notes. 2014;7:515.

4. Doege KW. Fibro-sarcoma of the mediastinum. Ann Surg. 1930;92:955-60.

5. Potter RP JR. Intrathoracic tumors: case report. Radiology. 1930;14:60-1.

6. Klemperer P, Coleman BR. Primary neoplasms of the pleura. A report of five cases. Am J Ind Med. 1992;22:1-31.

7. Fletcher $\mathrm{CD}$. The evolving classification of soft tissue tumours: an update based on the new WHO classification. Histopathology. 2006;48:3-12.

8. Hanau CA, Miettinen M. Solitary fibrous tumor: histological and immunohistochemical spectrum of benign and malignant variants presenting at different sites. Hum Pathol. 1995;26:440-9.

9. De Saint Aubain Somerhausen N, Rubin BP, Fletcher CD. Myxoid solitary fibrous tumor: a study of seven cases with emphasis on differential diagnosis. Mod Pathol. 1999;12:463-71.

10. Morimitsu Y, Nakajima M, Hisaoka M, Hashimoto H. Extrapleural solitary fibrous tumor: clinicopathologic study of 17 cases and molecular analysis of the p53 pathway. Apmis. 2000;108:617-25.

11. Mohajeri A, Tayebwa J, Collin A, Nilsson J, Magnusson L, von Steyern FV, et al. Comprehensive genetic analysis identifies a pathognomonic NAB2/ STAT6 fusion gene, nonrandom secondary genomic imbalances, and a characteristic gene expression profile in solitary fibrous tumor. Genes Chromosomes Cancer. 2013;52:873-86.

12. Robinson DR, Wu YM, Kalyana-Sundaram S, Cao X, Lonigro RJ, Sung YS, et al. Identification of recurrent NAB2-STAT6 gene fusions in solitary fibrous tumor by integrative sequencing. Nat Genet. 2013;45:180-5.

13. Gold JS, Antonescu CR, Hajdu C, Ferrone CR, Hussain M, Lewis JJ, et al. Clinicopathologic correlates of solitary fibrous tumors. Cancer. 2002;94:1057-68.

14. Hasegawa T, Matsuno Y, Shimoda T, Hasegawa F, Sano T, Hirohashi S. Extrathoracic solitary fibrous tumors: their histological variability and potentially aggressive behavior. Hum Pathol. 1999;30:1464-73.

15. Doyle LA, Vivero M, Fletcher CD, Mertens F, Hornick JL. Nuclear expression of STAT6 distinguishes solitary fibrous tumor from histologic mimics. Mod Pathol. 2014;27:390-5.

16. Witkin GB, Rosai J. Solitary fibrous tumor of the mediastinum. A report of 14 cases. Am J Surg Pathol. 1989;13:547-57.

17. Demicco EG, Park MS, Araujo DM, Fox PS, Bassett RL, Pollock RE, et al. Solitary fibrous tumor: a clinicopathological study of 110 cases and proposed risk assessment model. Mod Pathol. 2012;25:1298-306.

18. Scott K. Non-islet cell tumor hypoglycemia. J Pain Symptom Manage. 2009;37:e1-3.

19. Han G, Zhang Z, Shen X, Wang K, Zhao Y, He J, et al. Doege-Potter syndrome: a review of the literature including a new case report. Medicine (Balt). 2017;96:e7417

20. Dynkevich Y, Rother KI, Whitford I, Qureshi S, Galiveeti S, Szulc AL, et al. Tumors, IGF-2, and hypoglycemia: insights from the clinic, the laboratory, and the historical archive. Endocr Rev. 2013;34:798-826.

21. Gorden P, Hendricks CM, Kahn CR, Megyesi K, Roth J. Hypoglycemia associated with non-islet-cell tumor and insulin-like growth factors. N Engl J Med. 1981;305:1452-5.

22. Phillips LS, Robertson DG. Insulin-like growth factors and non-islet cell tumor hypoglycemia. Metabolism. 1993;42:1093-101.

23. Daughaday WH, Kapadia M. Significance of abnormal serum binding of insulin-like growth factor II in the development of hypoglycemia in patients with non-islet-cell tumors. Proc Natl Acad Sci U S A. 1989;86:6778-82.

24. Le Roith D. Seminars in medicine of the Beth Israel Deaconess Medical Center. Insulin-like growth factors. N Engl J Med. 1997;336:633-40.

25. Daughaday WH, Emanuele MA, Brooks MH, Barbato AL, Kapadia M, Rotwein $\mathrm{P}$, Synthesis and secretion of insulin-like growth factor II by a leiomyosarcoma with associated hypoglycemia. N Engl J Med, 1988;319(22):1434-40.

26. Marks AG, Carroll JM, Purnell JQ, Roberts Jr CT. Plasma distribution and signaling activities of IGF-II precursors. Endocrinology. 2011;152:922-30.

27. Ron D, Powers AC, Pandian MR, Godine JE, Axelrod L. Increased insulin-like growth factor II production and consequent suppression of growth hormone secretion: a dual mechanism for tumor-induced hypoglycemia. J Clin Endocrinol Metab. 1989;68:701-6.

28. van Doorn J, Hoogerbrugge CM, Koster JG, Bloemen RJ, Hoekman K, Mudde $\mathrm{AH}$, et al. Antibodies directed against the $\mathrm{E}$ region of pro-insulin-like growth factor-II used to evaluate non-islet cell tumor-induced hypoglycemia. Clin Chem. 2002;48:1739-50.

29. Bodnar TW, Acevedo MJ, Pietropaolo M. Management of non-islet-cell tumor hypoglycemia: a clinical review. J Clin Endocrinol Metab. 2014;99:713-22.

30. Zafar H, Takimoto $\mathrm{CH}$, Weiss $\mathrm{G}$. Doege-Potter syndrome: hypoglycemia associated with malignant solitary fibrous tumor. Med Oncol. 2003:20:403-8.

31. Prado F, Dos Ramos JP, Larrañaga N, Espil G, Kozima S. [Solitary fibrous tumor and Doege-Potter syndrome]. Medicina (B Aires). 2018;78:47-9. 